\title{
Do antipsychotic medications reduce or increase mortality in schizophrenia? A critical appraisal of the FIN-11 study D Cohen ${ }^{a, b}$, M De Hert ${ }^{c}$, CU Correll ${ }^{d, e}$
}

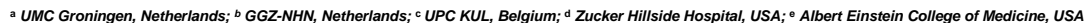

\section{Introduction}

Clozapine is effective in schizophrenia where other antipsychotics, typical or atypical, fail. Its haematological and metabolic side-effect require intensive monitoring and sometimes therapy.

\section{Study}

A large scale study in 66,881Finnish patients with schizophrenia (mean age 50 years) the mortality was during follow-up (mean FU: 7.8 years) was assessed (Tiihonen 2009).

\section{Results}

Out of the 66,881 patients, 19,735 (29.5\%) had died during the mean follow-up of 7.8 years.

Patients were divided according to AP-drug prescription (figure 1):

- 7 of the most prescribed AP drugs; other/rare; polypharmacy.

- Polypharmacy comprised 132,000 person years ( $40.3 \%$ ), more than the 7 most prescribed AP combined (125,298 person years)

The group 'Other/rare' comprised 70,520 person years, constituting the second largest group, bigger than any AP-drug on its own (i.e.: clozapine, the largest individual AP, comprised 32,000 person years).

The total number of deaths thus analysed was 4,100.

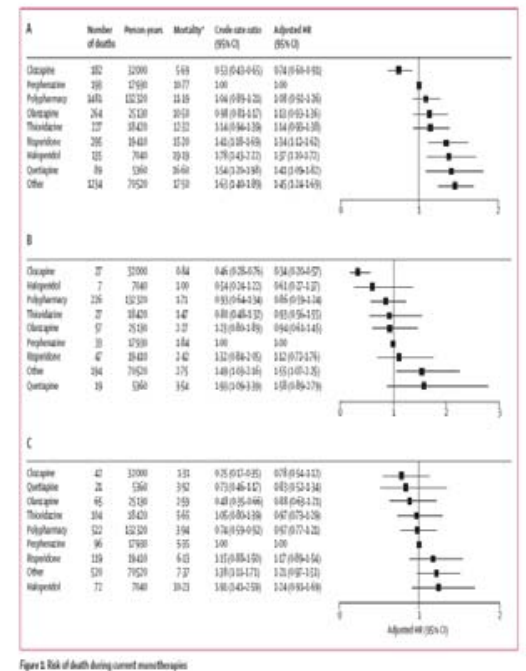

The consistent lowered mortality rate for clozapine was perhaps the most surprising finding of the study (figure 3)

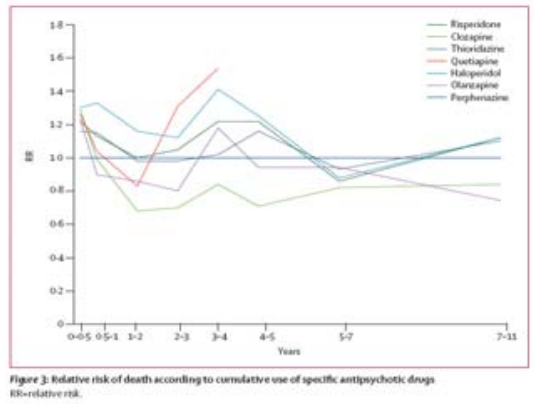

In the subgroup 'no AP' of 18,914 patients a total number of 8,277 patients ( $=43.8 \%$ ) died (figure 2 ).

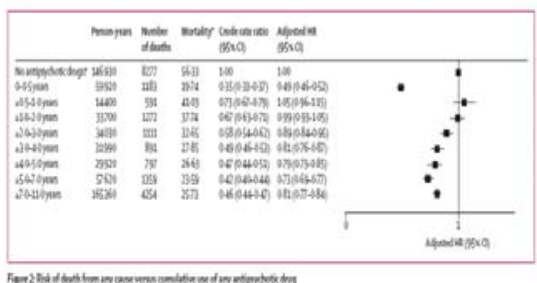

Discussion

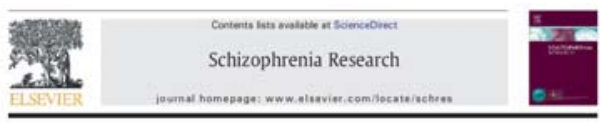

Do antipsychotic medications reduce or increase mortality in schizophrenia? A critical appraisal of the FIN-11 study Marc De Hert ${ }^{2 *}$, Christoph U. Correll ${ }^{\text {bec }}$, Dan Cohen ${ }^{\alpha}$

Death statistics: the missing $\mathbf{3 7 . 3 \%}$.

Deaths in whole study $19,735(100 \%)$ Deaths in 'no AP' $\quad 8,277(42 \%)$

Analysed $\quad 4,100(20.8 \%)$

Missing 7,358 (37.3\%)

Explanation: not provided. All deaths in hospital?

Comparison with Finnish mortality study.

$\begin{array}{lll} & \text { 'no AP' (Tiihonen) } & \text { Salokangas } \\ \text { Study duration } & 7.8 \text { years } & 3 \text { years } \\ \text { Total study mortality } & 43.8 \% & 5.6 \% \\ \text { Yearly mortality } & 5.6 \% & 1.7 \%\end{array}$

Systematic survivorship bias in clozapine users.

- Suicide risk is highest during the early phase of illness. However, clozapine is prescribed as a last resort to patients with a longer illness history who have not committed suicide.

Statistical adjustments can not account for this effect.

Method of analysis: cumulative use.

- Death was attributed both to current as to the previous AP drug, each proportional to treatment duration.

- Death on a current AP drug ' $X$ ' always contributed to the death incidence rate of all previously prescribed AP drugs.

Consequence of applied method of analysis:

- Inflation of attribution of death for all AP drugs, except for one: clozapine, which is the drug of last resort.

- The favourable outcome for clozapine may not reflect the reality, but rather the method of analysis.

Relation death and prescribed drug.

- The results are based on analysis of a minority of $20.8 \%$ of the deaths that occurred during the study.

- Even in this $20.8 \%$, the majority $(2,715)$ of the deaths occurred in the group of AP polypharmacy.

- The deaths rates are based on analysis of a minority of $13.8 \%(1,385)$ of the deaths that occurred during AP monotherapy.

- The group 'other/rare' was not rare at all. Actually, it was the one but biggest single group.

- Within the analysed sample, the two major groups of deaths were not analysed.

- The rate of untreated patients ('no AP') with an official diagnosis of schizophrenia during a mean follow-up of 7.8 years was high: 18,914 out of $66,881=28.3 \%$.

\section{Conclusion}

Methodology

The chosen method of analysis favours one and only one AP, clozapine, exactly the AP that systematically has the most favourable outcome in all applied analyses in this study.

\section{Representativeness:}

The great majority of the deaths that occurred during the followup, $79.2 \%$ (all patients except 4,100 ) if not $83.8 \%$ (all patients not on AP monotherapy) were not analysed.

Population with 'no AP':

- high percentage of $28.3 \%$ of untreated population with the official diagnosis of schizophrenia.

- high yearly mortality rate of $5.7 \%$.

Missing deaths

A substantial proportion of deaths $(7,358=37 . \%)$ is not accounted for.

Taking all of this into account, additional analyses of the Fin-11 data set are needed before the provided evidence and the proposed conclusions can be fully evaluated.

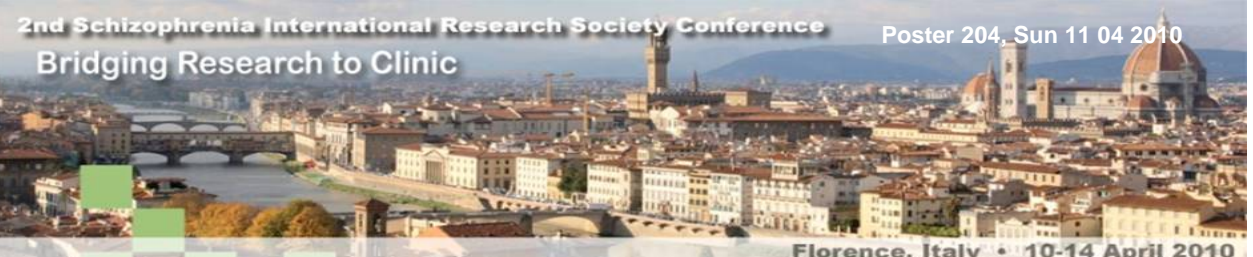

